\title{
Immediate Effects of a Novel Lumbar Support Device on Pain Modulation and Core Muscle Function in Patients with Chronic Non-Specific Low Back Pain: a Randomized Controlled Trial
}

\author{
D. Dissanguann ${ }^{1}$, P. Sitilertpisan ${ }^{1}$, L. H. Joseph², A. Paungmali ${ }^{1}$ \\ 1 Department of Physical Therapy, Faculty of Associated Medical Sciences, Chiang Mai University, Chiang Mai, \\ Thailand \\ 2 School of Sport and Health Sciences, University of Brighton, Eastbourne, East Sussex, U.K.
}

\author{
CORRESPONDING AUTHOR: \\ Aatit Paungmali \\ Department of Physical Therapy \\ Faculty of Associated Medical Sciences \\ Chiang Mai University \\ 110 Intawarorod Road \\ Sriproom, Moung \\ Chiang Mai 50200, Thailand \\ E-mail: aatit.p@cmu.ac.th \\ DOI: \\ 10.32098/mltj.03.2021.08
}

LEVEL OF EVIDENCE: 2B

\begin{abstract}
SUMMARY
Background. A novel lumbar support device to be used by people with chronic nonspecific low back pain (CNLBP) was designed, developed and tested in this trial. The study investigated the immediate effects of the novel lumbar support device on pain modulation and core muscle function in comparison to the traditional lumbar support among people with CNLBP.

Methods. This study was a single-blinded, repeated measures, randomized controlled trial with four parallel groups design. A total of 80 patients with CNLBP were randomly allocated using stratified block randomization by pain severity to one of the four groups: traditional lumbar support (TLS) as a control group $(\mathrm{N}=20)$, novel lumbar support with hot pack (NLSD + HP) $(\mathrm{N}=20)$, novel lumbar support with biofeedback device (NLSD $+\mathrm{BO})(\mathrm{N}=20)$, and finally the novel lumbar support with an in-built hot pack and biofeedback device (NLSD + HP with BO) as an experimental intervention group $(\mathrm{N}=20)$. Pain intensity (PI), tissue blood flow (TBF), cold pain threshold (CPT), hot pain threshold (HPT), thickness of transversus abdominis muscle (TrA), and lumbopelvic stability (LPS) were assessed at baseline and immediately post-intervention by a blinded assessor. A two-way repeated-measures ANOVA with post-hoc analysis was used to analyze the data.

Results. All 80 participants across the 4 groups had similar outcome measures at baseline $(\mathrm{p}>0.05)$. Among the participants in the NLSD + HP with BO (Group 4), a significant interaction was noticed in all of the primary outcomes including PI ( $p<$ 0.001), TBF ( $p<0.001)$, CPT ( $<<0.001)$, HPT ( $<<0.001)$, PPT ( $<<0.001), \operatorname{Tr} A$ thickness $(\mathrm{p}<0.001)$ and as well as on the secondary outcome lumbopelvic stability $(\mathrm{p}<0.001)$. Post hoc analysis showed that the results from the NLSD + HP with BO (Group 4) was superior than the TLS group in all of the primary and secondary outcomes $(\mathrm{p}<0.05)$.

Conclusions. The immediate effects of the NLSD + HP with BO demonstrated potential beneficial outcomes on PI, PPT, CPT, HPT, TrA and LPS in comparison to TLS among participants with CNLBP. Further clinical trial evaluating the long-term clinical effects of the novel lumbar support device is needed to understand the overall benefits of the device and its usefulness for people with CNLBP.
\end{abstract}

\section{KEY WORDS}

Back pain; core muscles; hot pack; lumbar support; rehabilitation. 
Low back pain (LBP) is a common musculoskeletal problem which leads to functional limitations and disability (1). Chronic low back pain (CLBP) is reported to affect the physical and mental health of the individuals (2). While there are several treatment strategies such as medication, surgery, acupuncture, manual therapy, physical exercises, heat and cold therapy reported for management of CLBP $(3,4)$, lumbar support (LS) is suggested as one among them to prevent and treat $\operatorname{LBP}(5,6)$. The mechanisms that have been proposed for the effectiveness of LS in the management of LBP are increase in intra-abdominal pressure, decrease in spinal load, increase in trunk stiffness, maintenance of correct posture and increase in proprioception are some of the proposed mechanisms behind how LS may help people with CLBP $(6,7)$. LS is reported to reduce the symptom of LBP and increase the patient's confidence to perform physical activities (8). Also, patients with LBP felt safer and more stable during physical activity when wearing lumbar support (9). Patients with CLBP also reported decreased pain and improved functional ability when wearing LS (5, 10). However, there is a concern that using a LS for a longer period may lead to a decrease in the abdominal and back muscle activity and trunk muscle weakness $(9,11)$. Therefore, LS seems to be effective when incorporating with the usual care of LBP management (12).

While some individuals may choose to use traditional lumbar support (TLS) to prevent or manage low back pain, the TLS only provides a passive support to the lumbosacral region. Thus, the passive therapeutic support provided by the TLS had raised some concerns that using TLS for a longer period of time might lead to a decrease in the abdominal and back muscle activity and reduced trunk muscle weakness $(9,11)$. A Cochrane systematic review from the neck and back group indicates that the TLS is not more effective than no intervention in preventing or treating CLBP (6). Perhaps, the TLS might be more effective when incorporating with exercises and usual care of CLBP management (12). Also, it could be possible that the current design of the TLS was not effective enough and a reconfiguration of the design of the lumbar support with additional therapeutic features was required. Therefore, a novel lumbar support device (NLSD) was designed and developed with additional therapeutic features and the current study was to report the investigation of the NLSD among individuals with CLBP. In addition to giving support to the lumbosacral region, the NLSD was designed with additional therapeutic features which includes a biofeedback mechanism to activate core muscles and with a provision to produce superficial moist heat therapy to back muscles. Thus, the patients could use the core muscle activation feedback in the NLSD to actively exercise their core muscles and as well as to apply a superficial heat therapy to the back muscles. Therefore, the main aim of the study was to investigate the immediate effects of NLSD in comparison to the TLS on pain modulation and core muscle function among patients with CLBP. The study hypothesis was that the NLSD would be superior to the TLS in improving pain modulation and core muscle function among people with CLBP.

\section{MATERIALS AND METHODS}

The current study was a single-blinded, repeated measures, randomized controlled trial with four parallel groups. The study protocol was registered in the randomized control trial registry (TCTR20190905002) and approved by the institutional ethical committee according to the standards of the Declaration of Helsinki (AMSEC60X028). All participants were informed about the study and signed a written informed consent prior to their participation in the study. All authors have followed the international ethical principles and the ethical standards of the Muscle, Ligaments and Tendons Journal (13).

\section{Randomization and allocation}

Participants were randomly allocated (1:1:1:1 ratio) to one of four intervention groups 1) Group 1- TLS group, 2) Group 2 - NLS with hot pack feature (NLS + HP), 3) Group 3 NLS with biofeedback core muscle activator (NLS + BO) and 4) Group 4 - NLSD which had both the features of hot pack and biofeedback core muscle activator (NLSD + $\mathrm{HP}$ with $\mathrm{BO}$ ). The randomization was stratified by severity of back pain (i.e., pain intensity). An internet randomized scheme generator which generated block randomization with a random sequence of permuted blocks of 8 was used for allocating the participants for the intervention groups. An independent research staff undertook randomization and allocation of the participants in the trial.

\section{Allocation concealment}

The group assignment number was written on a piece of paper and placed in an opaque envelope and sealed. The member of the staff who conducted these processes did not have any roles in data collection or intervention assignment. The opaque sealed envelopes were opened after completion of baseline assessment.

\section{Participants}

The study was conducted in a neuromuscular and pain research unit at the physiotherapy department in a universi- 
ty institutional setting during January and December 2019. A total of 80 participants both males and females aged between 20-55 years with non-specific chronic low back pain (NSCLBP) were recruited into the study. The study details and participant recruitment procedures were advertised through community centers and Facebook, and advertisement posters were placed in the hospitals, physical therapy clinics, and university campus to facilitate recruitment. The inclusion criteria were: 1 ) pain in the area between $12^{\text {th }}$ rib to gluteal fold for more than three months, 2) body mass index (BMI) between $18.5-30 \mathrm{~kg} / \mathrm{m}^{2}$, and 3) willing to participate in the study voluntarily. Participants excluded were those that had any of the following: 1) referred pain or numbness at lower limbs, 2) impaired sensation at the body and lower limbs, 3) history of past surgery of spine or lower extremities, 4) history of injury from an accident in the previous three months, 5) structural deformities of the spine, 6) pregnancy, 7) specific spinal disorders or nerve root compression, 8) inflammation or infection at spine and back, 9) severe medical conditions such as cardiovascular disease, renal failure, hypertension, diabetes, and 10) those who received any pain-relieving medication in the previous three months. Figure 1 shows the flow of the study recruitment.

\section{Interventions}

A qualified therapist experienced in the field of musculoskeletal physiotherapy provided the intervention. Blinding of participants was not possible due to the nature of the interventions. Participants were advised to inform if they would experi-
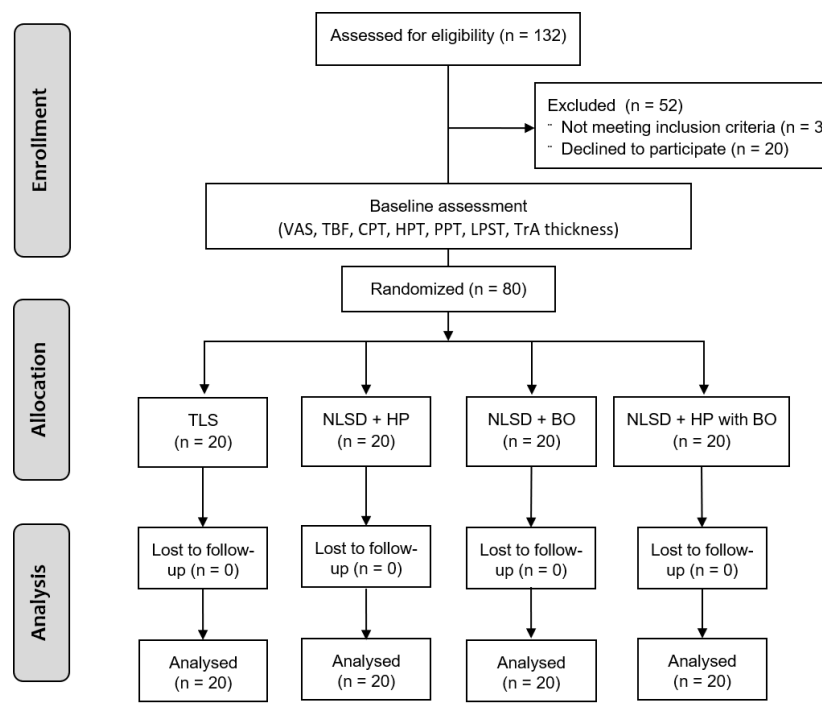

Figure 1. CONSORT flow diagram of participant recruitment and allocation in the study. ence any discomfort during the interventions and were allowed to leave the experiment if the participants requested to leave the study due to any personal reasons or reported worsening of symptoms. Any adverse events or any incidents of reported discomfort during the intervention period were recorded.

\section{Group 1: Traditional lumbar support (TLS) as Control group}

Participants received a TLS (model: LS, Manufacturer: VR Support Limited, Thailand) which fitted to the body size of each participant. Two sizes of lumbar support (i.e., medium and large) were used for the interventions generally according to the hip sizes of the participants. The participants were taught to wear lumbar support around the lumbopelvic region (the upper edge of lumbar support is just below the $12^{\text {th }}$ ribs) firmly. The participants had worn a traditional lumbar support and were asked to complete 4 rounds of standing for 4 minutes and had a rest by sitting for 1 minute. The standing and sitting positions were chosen to represent the functional activities and common gestures of working in daily life. As part of the intervention to measure the immediate effects of the lumbar support, the participants were instructed to wear the TLS for 20 minutes.

\section{Group 2: Novel lumbar support device with in-built hot pack (NLSD + HP)}

The selection of the sizes of the lumbar support, application technique and the familiarization procedure were performed similar to the control group. However, the NLSD + HP used in the Group 2 consisted of an additional feature to provide superficial heat therapy through a hot pack feature designed in the lumbar support. While the participants wore the lumbar support, the participants received superficial heat therapy to back muscles from the $\mathrm{NLSD}+\mathrm{H}$ for 20 minutes.

\section{Group 3: Novel lumbar support device with in-built biofeedback device (NLSD + BO)}

The familiarization protocol and application techniques were similar to group 1 and 2. The NLSD + BO was designed with an additional feature consisting of a mechanism that provided a visual biofeedback whenever the participants successfully engaged the core muscles. The validity and reliability of the feedback sensor to facilitate the core muscles activation was tested and established prior to the intervention (14). The feedback sensor was a biofeedback device that was developed using a pressure sensor. The feedback sensor was designed with a mechanism to give both audio and visual biofeedback 
to the participants every time when they contracted the core muscles during ADIM. The participants could select whether they would like to receive either an audio biofeedback through a beeping sound or a visual feedback through a blinking light while they tried to contract the core muscles. With the feedback sensor fixed within the lumbar support, the participants were taught to perform core muscle activation by doing an abdominal drawing-in maneuver (ADIM) and the biofeedback device facilitated the maneuver by providing a visual and auditory feedback to the participants to engage with subsequent core muscle activation. While wearing the lumbar support, the participants were instructed to perform ADIM in the standing position and hold it for 10 seconds/ time, 20 times/set 4 sets and to rest for 1 minute by siting in between the sets. The whole session lasted for 20 minutes.

\section{Group 4: Novel lumbar support with in-built hot pack \& biofeedback (NLSD + HP with BO)}

The application techniques and familiarization procedures were performed similar to the above groups. The NLSD + $\mathrm{HP}$ with $\mathrm{BO}$ was designed with two additional features which included a mechanism to produce superficial heat therapy and had a feature to provide a visual feedback to participants to exercise the core muscles. While wearing the lumbar support, the participants superficial heat therapy to back muscles and also performed ADIM holding it for 10 seconds/time, 20 times/set, 4 sets with 1-minute rest by sitting in between the sets. The duration of the whole intervention was 20 minutes.

\section{Outcome measures}

An independent assessor measured all the outcomes of the trial. The outcome measures were measured at baseline and immediately after 20 minutes of the intervention in each group. An intra-rater reliability was established prior to the enrollment of participants and data collection, with an acceptable value of agreement greater than $80 \%$ and intraclass correlation coefficients (ICCs) ranged from 0.87-0.99 for all measures. Pain modulation was measured as one of the primary outcomes through evaluating changes in the pain intensity (PI), pressure pain threshold and thermal pain threshold. In addition, thickness of transversus abdominis muscle and the tissue blood flow were measured as additional primary outcomes. Lumbopelvic stability test was used to evaluate the stability of the lumbopelvic region as a secondary outcome. The procedure for measuring pain modulation was conducted as per previously established protocol $(15,16)$. Visual analog scale (VAS) was used to assess pain intensity. The participants rated their pain intensity (PI) on a horizontal line in a 100 millimeters length scale which says "no pain" on the left hand and "pain as bad as possible" on the right hand of the scale (17). Pressure pain threshold (PPT) was assessed by using pressure algometer (Somedic Production, Algometer type II, Sweden). Pressure was applied perpendicularly to the skin at constant speed of $40 \mathrm{kPa} / \mathrm{s}$. The participants pressed a button to inform the sensation changing from the pressure to pain by pressing a button. PPT was randomly measured over the standard fixed point (facet joints) of L4-L5 on both sides. PPT was assessed 3 times with 30-sec resting between trials and mean of the 3 trials will be used for analysis (18). The thermal pain threshold was measured by a Thermal Sensory Analyzer (Medoc Ltd., Neuro Sensory Analyzer Model TSA-II, Israel) for cold pain threshold (CPT) and heat pain threshold (HPT). A $5-\mathrm{cm}^{2}$ thermode was directly applied to the skin over the L4-5 interspinous space. The initial temperature was set at 32 ${ }^{\circ} \mathrm{C}$ with the rate change 1 degree Celsius/second for heat pain and 2 degrees Celsius/second for cold pain (18). The cut off temperature was set at $0{ }^{\circ} \mathrm{C}$ for cold pain threshold and 50 ${ }^{\circ} \mathrm{C}$ for heat pain threshold for preventing any possible tissue damage. The participants were instructed to press the button when they felt the sensation changing from heat or cold to pain. Thermal pain threshold was assessed 3 times and mean of the 3 trials was used for analysis.

Real-time ultrasound imaging was used to evaluate the muscle function of the transversus abdominis muscle (TrA) which is an important core stabilizing muscle. The muscle function of the TrA was measured as per an established protocol (19). An ultrasound scanner in B-mode with $5 \mathrm{MHz}$ with curvilinear transducer was used to assess the muscle thickness of $\operatorname{TrA}$ (figure 2). The participants were positioned in crooked lying with a pillow under their head and knees. The transducer was placed at a point $2.5 \mathrm{~cm}$ in the anteromedial region in the midpoint between lower rib and iliac crest on midaxillary line in transverse plane and the image was collected at the end of exhalation. Thickness of $\operatorname{Tr} A$ was randomly measured on both sides and the mean thickness of three measurements was calculated for analysis. The tissue blood flow (TBF) was measured using a Doppler blood flow meter (Moor instruments DRT4, UK) as per an established protocol (16). The participants lied in prone position with arm by side. The electrode of the Doppler blood flow meter was placed over a standard fixed point on the tenderest place over the lumbar paravertebral muscle between the first and fifth lumbar vertebral region. The TBF was recorded every minute for a period of 5 minutes and the mean value was used for analysis. In addition, stability of the lumbopelvic region (LPS) was evaluated as a secondary outcome measure using the lumbopelvic stability test. With the participant in supine lying position with knee flexed to 70 degrees, a pressure biofeedback unit was pumped to $40 \mathrm{mmHg}$ and placed under the lumbar spine between 


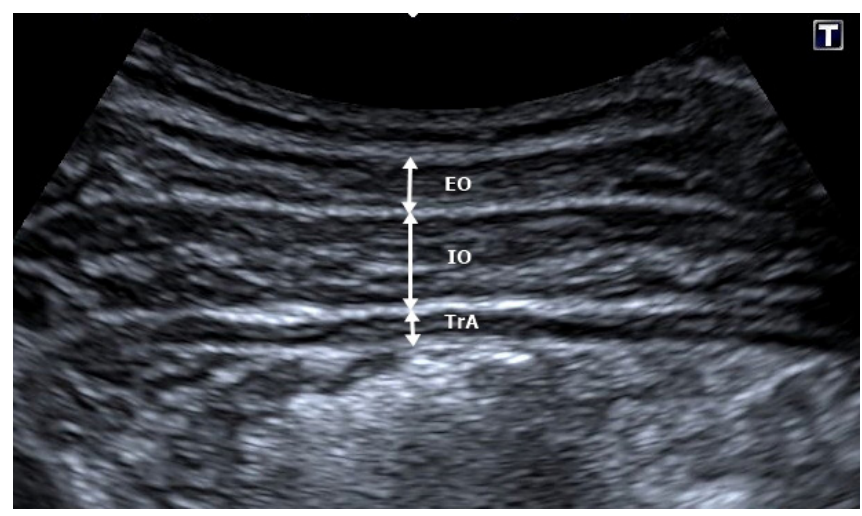

Figure 2. Ultrasound image of the Transversus abdominis muscle (TrA), Internal oblique (IO) and External oblique (EO).

the lumbar and sacral region (L2-S1). The participants performed a unilateral leg lift in the sagittal plane followed by progressive levels of seven lumbopelvic stability tests as per the test protocol (16). The participants were asked to maintain the pressure within $40 \pm 4 \mathrm{mmHg}$ in the pressure biofeedback device while progressing to different levels of test. The test was stopped when the participants were not able to hold $40 \pm 2 \mathrm{mmHg}$ in the biofeedback device and the test level was noted.

\section{Statistical analysis}

The sample size was calculated with a $G$ *power program for a significant alpha level of 0.05 and a power analysis of 0.8 with an estimated effect size of 0.54 . The collected data were analyzed for normal distribution using the Kolmogorov Smirnov test. A two-way repeated-measures ANOVA (4 conditions $\times 2$ times) was used to consider the interaction effects and the main effects of the experimental conditions and time. A post-hoc analysis was conducted, and the level of significance was set as $(\mathrm{p}<0.05)$. The percentage change in all variables was measured by calculating the difference between pre- and post-intervention changes divided by 100 . The estimated effect size was calculated using partial eta square $\left(\eta_{\mathrm{p}}{ }^{2}\right)$. An effect size was interpreted as following: small effect (0.2), medium effect (0.5), and large effect (0.8) (20).

\section{RESULTS}

A total of 80 participants ( 33 males and 47 females) with CNSLBP participated in this study with a mean age (41.3 \pm 9.1) (years) and mean BMI score $(23.5 \pm 13.2)(\mathrm{kg} /$ $\mathrm{m}^{2}$ ). The mean onset of low back pain among the participants was $35.9 \pm 38.8$ months and they reported Oswestry Disability Index mean score of $18.7 \pm 10.6$ (\%). The characteristics of the participants allocated to the four groups were shown in the table I. There were no significant differences between the study groups in terms of gender, age, body mass index (BMI), the onset of LBP, and disability score at the baseline.

There were no significant differences in any of the variables at the baseline between the study groups $(p>0.1)$. Table II shows the two-way repeated measure ANOVA results on the interaction effect between groups and time in all variables $(\mathrm{p}<0.05)$. The participants in the TLS (Group 1) showed significant interaction only in the PI $(\mathrm{p}<0.001)$, and PPT $(\mathrm{p}<0.01)$ in comparison to baseline measures. The results showed that the participants in the NLSD + HP (Group 2) showed significant interaction in PI ( $\mathrm{p}$ $<0.001)$, TBF $(\mathrm{p}<0.001)$, CPT $(\mathrm{p}<0.001)$ and PPT (p $<0.001$ ), however no interaction was noticed in the $\operatorname{Tr} A$ thickness $(p>0.05)$ and stability of the lumbopelvic region ( $p>0.05$ ). In comparison to the baseline values, the results showed that the participants in the NLSD + BO (Group 3 ) had significant interaction in PI ( $p<0.001)$, TBF ( $p<$ $0.05)$, CPT $(\mathrm{p}<0.05)$, PPT $(\mathrm{p}<0.001), \operatorname{Tr} \mathrm{A}$ thickness $(\mathrm{p}$ $<0.001)$, lumbopelvic stability $(\mathrm{p}<0.001)$ except HPT $(\mathrm{p}>0.05)$. Finally in the NLSD + HP with BO (Group 4), a significant interaction was noticed in all of the primary outcomes including PI $(\mathrm{p}<0.001)$, TBF $(\mathrm{p}<0.001)$, CPT $(\mathrm{p}<0.001)$, HPT $(\mathrm{p}<0.001)$, PPT $(\mathrm{p}<0.001)$, TrA thickness $(\mathrm{p}<0.001)$ and as well as on the secondary outcome lumbopelvic stability $(\mathrm{p}<0.001)$. Post hoc analysis showed

Table I. Characteristics of the study participants shown as mean \pm standard deviation (SD) across the four groups.

\begin{tabular}{|c|c|c|c|c|c|}
\hline Variables/ Group & $\begin{array}{l}\text { TLS } \\
(n=20)\end{array}$ & $\begin{array}{l}\text { NLSD + HP } \\
(n=20)\end{array}$ & $\begin{array}{l}\text { NLSD + BO } \\
(n=20)\end{array}$ & $\begin{array}{l}\text { NLSD + } \\
\text { HP with BO } \\
(n=20)\end{array}$ & p-value \\
\hline Gender $(\mathrm{M} / \mathrm{F})$ & $8 / 12$ & $10 / 10$ & $6 / 14$ & $9 / 11$ & 0.614 \\
\hline Age (years) & $40.5 \pm 9.99$ & $41.45 \pm 9.93$ & $40.45 \pm 7.8$ & $43.05 \pm 8.82$ & 0.789 \\
\hline BMI $\left(\mathrm{kg} / \mathrm{m}^{2}\right)$ & $23.46 \pm 3.96$ & $23.96 \pm 2.86$ & $22.55 \pm 3.33$ & $24.24 \pm 2.75$ & 0.384 \\
\hline Onset of LBP (months) & $24.75 \pm 22.87$ & $31.05 \pm 34.72$ & $48.1 \pm 59.53$ & $40.0 \pm 38.42$ & 0.302 \\
\hline
\end{tabular}


Table II. Data of all variables and mean difference values are shown as mean (SD).

\begin{tabular}{|c|c|c|c|c|c|c|c|}
\hline \multirow[t]{2}{*}{ Outcomes } & & \multicolumn{2}{|c|}{ Groups } & \multirow[b]{2}{*}{$\begin{array}{l}\text { NLSD + BO } \\
(n=20)\end{array}$} & \multirow[b]{2}{*}{$\begin{array}{l}\text { NLSD + HP } \\
\text { with BO } \\
(\mathrm{n}=20)\end{array}$} & \multicolumn{2}{|c|}{ Interaction effect } \\
\hline & & $\begin{array}{l}\text { TLS } \\
(n=20)\end{array}$ & $\begin{array}{l}\text { NLSD + HP } \\
(n=20)\end{array}$ & & & p-value & $\eta_{p}^{2}$ \\
\hline VAS & Pre & 30.65 & 36.25 & 34 & 33 & 0.001 & 0.2 \\
\hline \multirow[t]{5}{*}{$(\mathrm{mm})$} & & $(22.74)$ & $(18.37)$ & $(22.49)$ & $(18.49)$ & & \\
\hline & Post & $20.9^{* * * *}$ & $17.4^{* * * *}$ & $23.35^{* \ldots *}$ & $15.65^{\text {*** }}$ & & \\
\hline & Mean Diff & $-9.75^{b, d}$ & $-18.85^{\mathrm{a}, \mathrm{c}}$ & $-11^{b, d}$ & $-17.4^{\mathrm{a}, \mathrm{c}}$ & & \\
\hline & $(95 \% \mathrm{CI})$ & $(-13.37,-6.13)$ & $(-22.56,-12.14)$ & $(-14.98,-7.02)$ & $(-21.07,-13.73)$ & & \\
\hline & $\begin{array}{l}\text { Percentage } \\
\text { Change }(\%)\end{array}$ & $\begin{array}{l}-43.47 \\
(32.76) \\
\end{array}$ & $\begin{array}{l}-57.36 \\
(22.24) \\
\end{array}$ & $\begin{array}{l}-36.56 \\
(20.84) \\
\end{array}$ & $\begin{array}{l}-61.72 \\
(26.34) \\
\end{array}$ & & \\
\hline $\mathrm{TBF}$ & Pre & 10.13 & 9.94 & 10.07 & 10.41 & $<0.001$ & 0.71 \\
\hline \multirow{3}{*}{ (flux/min) } & Mean Diff & $2.03^{b, d}$ & $31.12^{a, c}$ & $3.93^{b, d}$ & $33.04^{\mathrm{a}, \mathrm{c}}$ & & \\
\hline & $(95 \% \mathrm{CI})$ & $(0.52,0.72)$ & $(23.66,38.58)$ & $(-0.79,8.64)$ & $(26.11,39.96)$ & & \\
\hline & $\begin{array}{l}\text { Percentage } \\
\text { Change }(\%)\end{array}$ & $\begin{array}{l}24.24 \\
(34.7) \\
\end{array}$ & $\begin{array}{l}341.99 \\
(208.3) \\
\end{array}$ & $\begin{array}{l}47.07 \\
(118.34)\end{array}$ & $\begin{array}{l}368.08 \\
(234.71)\end{array}$ & & \\
\hline $\mathrm{PPT}$ & Pre & 416.09 & 452.75 & 445.62 & 457.35 & $<0.001$ & 0.48 \\
\hline \multirow[t]{4}{*}{$(\mathrm{kPa})$} & & $(136.92)$ & $(183.74)$ & $(179.35)$ & $(195.92)$ & & \\
\hline & Post & $441.78^{* *}$ & $562.73^{* * * * x}$ & $500.97^{* * *}$ & $569.79^{\text {*k:k }}$ & & \\
\hline & & $(132.44)$ & $(207.32)$ & $(185.33)$ & $(199.41)$ & & \\
\hline & Mean Diff & $25.69^{b, c, d}$ & $109.98^{\mathrm{a}, \mathrm{c}}$ & $55.35^{\mathrm{a}, \mathrm{b}, \mathrm{d}}$ & $112.44^{\mathrm{a}, \mathrm{c}}$ & & \\
\hline \multirow{4}{*}{$\left({ }^{\circ} \mathrm{C}\right)$} & & $(2.78)$ & $(1.42)$ & $(2.06)$ & $(1.4)$ & & \\
\hline & Mean Diff & $-0.28^{b, d}$ & $-2.16^{a, c}$ & $-0.78^{b, d}$ & $-2.27^{a, c}$ & & \\
\hline & $(95 \% \mathrm{CI})$ & $(-0.43,0.98)$ & $(-2.87,-1.45)$ & $(-1.49,-0.08)$ & $(-2.98,-1.56)$ & & \\
\hline & $\begin{array}{l}\text { Percentage } \\
\text { Change }(\%)\end{array}$ & $\begin{array}{l}-13.57 \\
(30.09) \\
\end{array}$ & $\begin{array}{l}-66.08 \\
(36.03) \\
\end{array}$ & $\begin{array}{l}-36.19 \\
(41.8) \\
\end{array}$ & $\begin{array}{l}-62.19 \\
(38.35) \\
\end{array}$ & & \\
\hline HPT & Pre & 43.49 & 43.91 & 45.16 & 44.35 & $<0.001$ & 0.59 \\
\hline \multirow[t]{5}{*}{$\left({ }^{\circ} \mathrm{C}\right)$} & & $(3.46)$ & $(2.8)$ & $(2.83)$ & $(3.43)$ & & \\
\hline & Post & 43.72 & 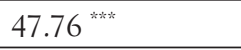 & 45.02 & $47.06^{* * * *}$ & & \\
\hline & & $(3.36)$ & $(2.03)$ & $(3.02)$ & $(2.51)$ & & \\
\hline & Mean Diff & $0.23^{b, d}$ & $3.85^{a, c, d}$ & $-0.14^{b, d}$ & $2.71^{\mathrm{a}, \mathrm{b}, \mathrm{c}}$ & & \\
\hline & $(95 \% \mathrm{CI})$ & $(-0.4,0.87)$ & $(3.21,4.48)$ & $(-0.78,0.49)$ & $(2.08,3.35)$ & & \\
\hline
\end{tabular}




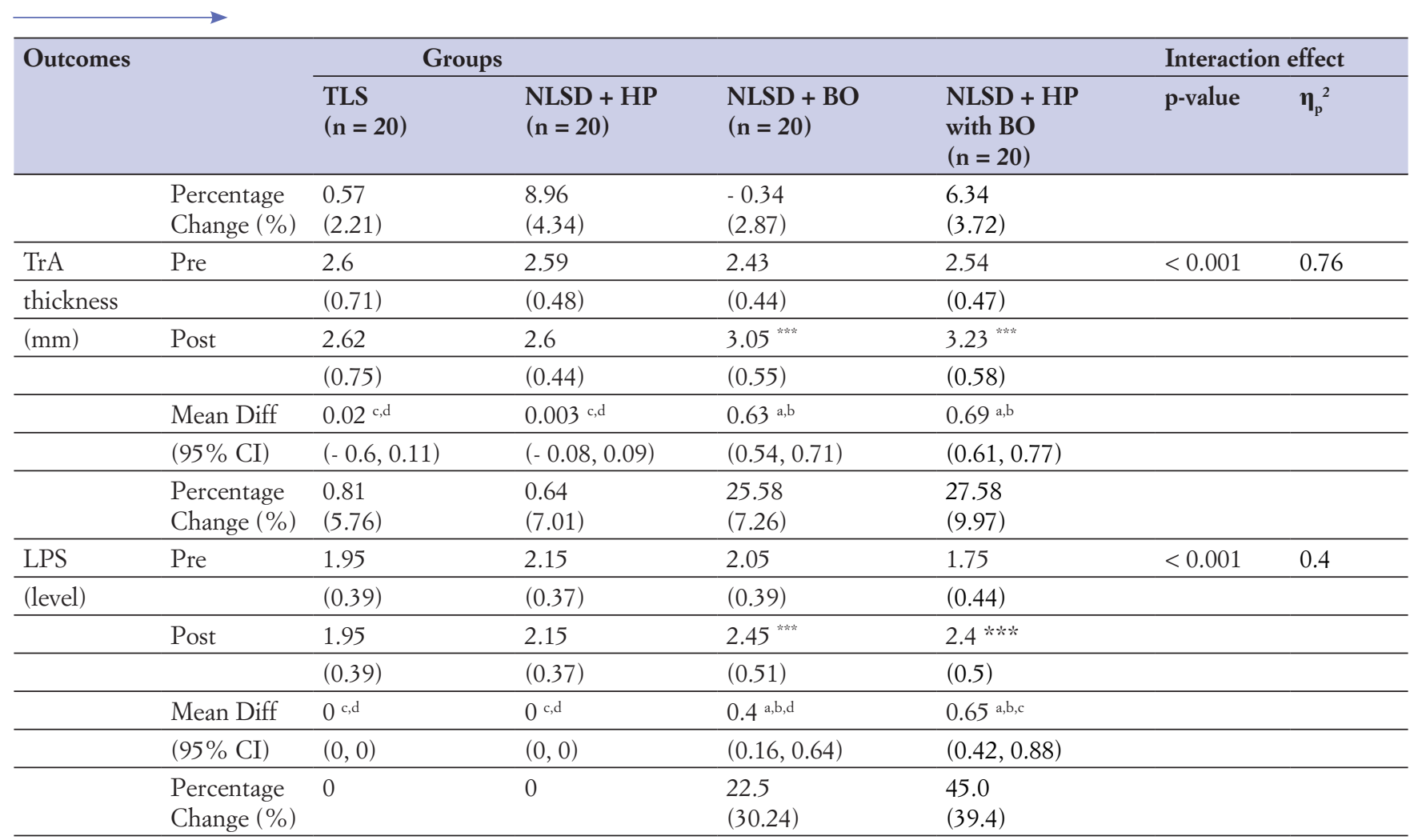

No significant differences in the baseline data among 4 conditions $(\mathrm{p}>0.05)$. Significant differences between pre-post $\left({ }^{*} \mathrm{p}<0.05, * * \mathrm{p}<0.01, * * * \mathrm{p}<0.001\right)$. ${ }^{\mathrm{a}}$ Significant difference between control $(\mathrm{p}<0.05) .{ }^{\mathrm{b}}$ Significant differences between LS + H P $(\mathrm{p}<0.05)$. ${ }^{\mathrm{c}}$ Significant differences between LS + CSE $(\mathrm{p}<0.05)$. ${ }^{\mathrm{d}}$ Significant differences between LS $+\mathrm{HP}+\mathrm{CSE}(\mathrm{p}<0.05) . \eta_{\mathrm{p}}{ }^{2}=$ Partial eta-squared. ES = Effect size (Cohen's $\mathrm{f}$ score).

that the results from the NLSD + HP with BO (Group 4) was superior than the TLS group in all of the primary and secondary outcomes. Also, the post hoc analysis showed that the NLSD + HP with BO (Group 4) results were superior to the NLSD + HP (Group 2) in the outcomes such as HPT, TrA thickness and stability of lumbopelvic region ( $\mathrm{p}$ $<0.05$ ) and as well as superior to the NLSD + BO (Group $3)$ in all of the primary $(\mathrm{p}<0.05)$ and secondary outcomes $(\mathrm{p}<0.05)$ except $\operatorname{Tr} A$ thickness $(\mathrm{p}>0.05)$. Figure 3 showed the percentage change for all outcome measures.

\section{DISCUSSION}

The current study investigated the immediate effects of NLSD + HP with BO on PI, TBF, PPT, HPT, CPT, TrA thickness and LPS in comparison to TLS among patients with CNLBP. The results of the study supported the hypothesis that the NLSD + HP with BO in comparison to TLS had superior therapeutic effects in all the primary outcomes (PI, TBF, PPT, HPT, CPT, TrA thickness) and secondary outcome (LSP) among CNLBP patients. TLS has some significant challenges in terms of application to clinical practice for management of CNLBP. Current state of scientific evidence questions the effectiveness of the TLS and raises concern on the use of lumbar support for the prevention and management of low back pain (6). Also, prolonged use of TLS is reported to cause trunk muscle weakness and decreased trunk muscle activity ( 9 , 11). While there are different brands of lumbar support is available, they all were designed to provide a passive support to the lumbar region. Therefore, it was decided to redesign a lumbar support with built in additional features such as superficial heat therapy and a biofeedback to exercise the core muscles. Thus, two more study groups NLSD + HP (Group 2) and NLSD + BO (Group 3) were added as additional comparative groups in this trial to study and evaluate the effects of having the superficial heat therapy and biofeedback mechanism in the lumbar support. Finally, NLSD + HP with BO (Group 4) was added and studied as the experimental intervention group to evaluate the combined effects of adding superficial heat therapy and biofeedback mechanism in the lumbar support. Prior to the initiation of the current trial, the design and function of the novel lumbar support device was tested and proven 


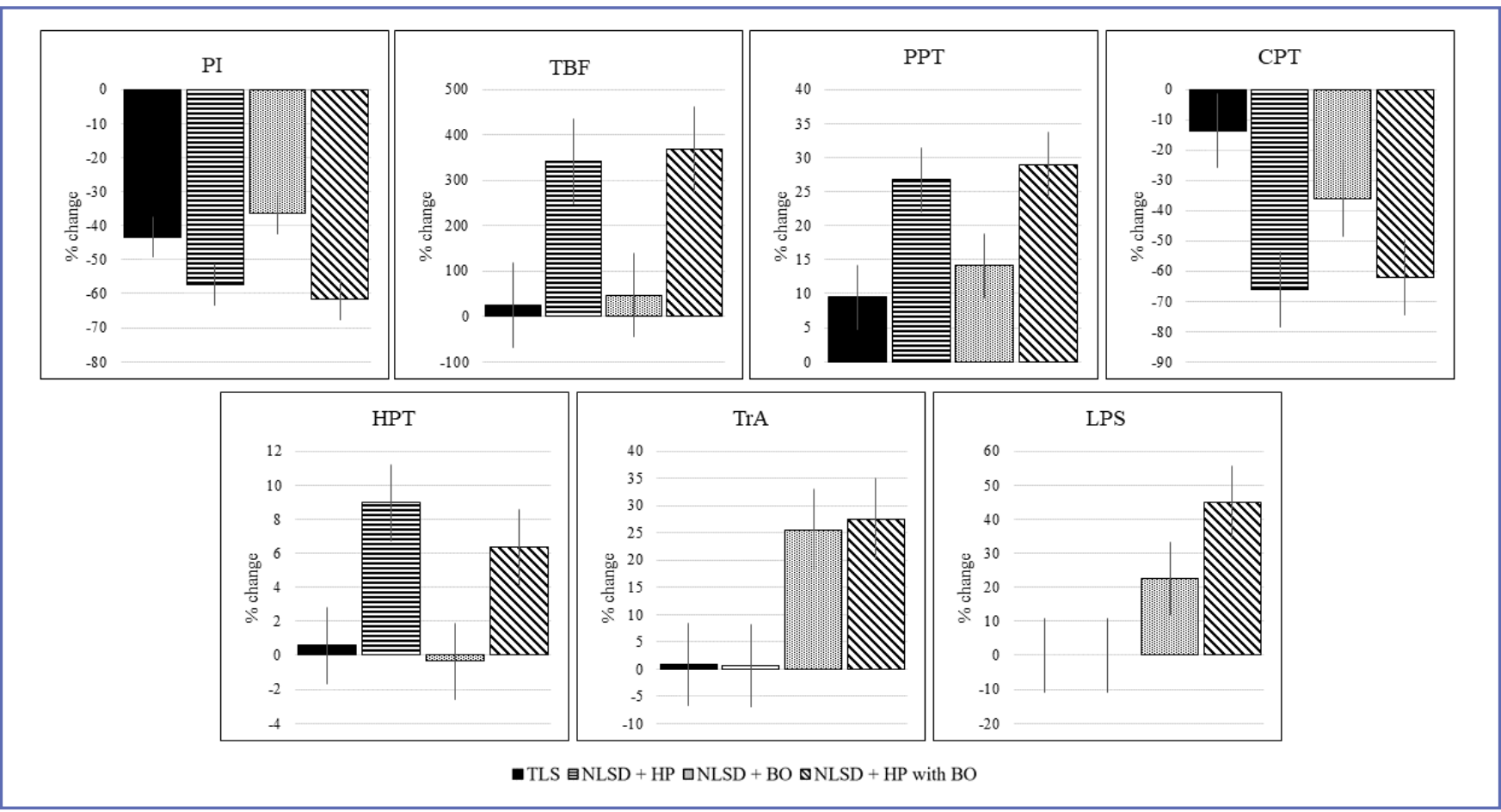

Figure 3. The percentage change for the primary and secondary outcomes. Traditional lumbar support (TLS): filled column, novel lumbar support with hot pack (NLSD + HP): striped column, novel lumbar support with biofeedback device (NLSD + BO): dotted column, novel lumbar support with hot pack and biofeedback device (NLSD + HP with BO): twill column. PI: pain intensity, TBF: tissue blood flow, PPT: pressure pain threshold, CPT: cold pain threshold, HPT: heat pain threshold, TrA: thickness of transversus abdominis muscle, LPST: lumbopelvic stability test.

to have reliable and valid features (14). Thus, the results of the study validated the design features of the NLSD + HP with $\mathrm{BO}$ and supported that the inclusion of additional novel features in the device such as superficial heat therapy with a biofeedback mechanism to engage core muscles produced desirable benefits to the CNLBP patients.

The results suggested that wearing the NLSD + HP with BO for 20 minutes induced an immediate physiological change as observed by an increase in the TBF lumbar region. Increase in TBF is suggested to promote the healing process by supplying more oxygen and nutrients to the site of injury and removes the irritant substances (21). Therefore, the magnitude of change in the TBF reported in the study might have positive implications for CNLBP patients. The increase in TBF was found to be higher in the group which had NLSD + HP with BO and the effect was clinically significant with a larger effect size (partial eta-squared $\left(\eta_{p}^{2}\right)$ - 0.7) when compared to the other three groups. In clinical practice, quantitative sensory testing (QST) is used to evaluate hypersensitivity and hyperalgesia which are associated with long term pain conditions such as CNLBP (22). Therefore, QST (PPT, HPT and CPT) was used as a standardized outcome measure along with PI to evaluate the effects on pain modulation $(17,22)$. In clinical setting, the practitioners might be able to understand the effects of an intervention by monitoring improvement in the pain reported by patients (15), hence pain modulation was observed as a primary outcome measure through evaluating QST along with PI. Besides the increase in the TBF, the results showed that NLSD + HP with BO had immediate effects on pain modulation. The results showed that the percentage increase of PPT was observed to be higher in the NLSD + HP group $(28.92 \%)$ compared to the TLS group (9.51\%). As PPT has been suggested to be most predictive of CNLBP (23), the clinically meaningful change in PPT among participants who used NLSD $+\mathrm{HP}$ with $\mathrm{BO}$ may be considered as a useful finding in this study. Besides increasing tolerance of mechanical pain, CPT and HPT also showed significant positive changes in the NLSD + HP with BO group where by the CPT decreased by approximately $62.2 \%$ and HPT improved by $6 \%$. In addition, the PI also a clinical meaningful reduction $(61.7 \%)$ in Group 4 in comparison to other three groups. Superficial heat therapy results in pain relief, 
reduced muscle stiffness, and increased flexibility among LBP patients (24). The novel lumbar support device was designed to provide superficial heat therapy to back muscles which could explain the overall superior effects on pain modulation observed in the NLSD $+\mathrm{HP}$ with BO group when compared to the TLS group.

Deeper abdominal muscle such as $\operatorname{Tr} A$ provides stability to the lumbopelvic region. Also, $\operatorname{Tr} A$ has been reported to have impairment among people with LBP (25). Therefore, thickness of the TrA muscle was evaluated to measure the changes in TrA muscle activity and LPS was evaluated as a secondary outcome measure in the study. When compared to the TLS group, the participants in the NLSD $+\mathrm{HP}$ with $\mathrm{BO}$ group showed a higher increase in both the TrA muscle activity and LPS with a percentage change of $27.5 \%$ and $45 \%$ respectively. An evidence suggests that lumbar support may positively affect trunk motion as it reduces trunk motion for flexion- extension and lateral bending (7). However, there is no evidence to suggest that this can increase TrA muscle activity and LPS. Currently, there are no data from previous literature available to compare the effects of lumbar support on LPS and $\operatorname{TrA}$ thickness. The novel lumbar support device had a design feature of a biofeedback mechanism to support and assist the participants to exercise the deeper core muscles. Prior to the current study, the reliability and validity of the biofeedback mechanism in the novel lumbar support for activation of core muscle ( $\operatorname{Tr} A)$ was established (14). Perhaps, the changes observed on LSP and TrA muscle activity might be related to the fact that the participants in the NLSD + HP with BO group used the biofeedback device and exercised their core muscles while they wear the novel lumbar support. Also, it is possible that the increase in the TrA muscle activity and LPS might be related with the pain modulation effects reported among the study participants. Further studies are warranted to understand the underlying mechanism and effects of the NLSD + HP with BO on trunk stability.

This study may have some limitations. First, the study investigated only the immediate effects of the novel lumbar support device to establish its potential mechanisms of action on pain modulation and core muscle function. Nevertheless, the study on the immediate effects was warranted to establish the performance of the novel lumbar support device and as well the findings of the current study was used as a catalyst to plan and conduct a long-term trial. The NLSD + HP with BO provided a superficial heat therapy to the back muscles for about 20 minutes which might have caused changes in the tissue temperature of skin and muscles. As the post study measurements were conducted immediately after the heat therapy application, the change in the skin temperature could be a potential confounder to the thermal threshold measurements. However, having the TLS group as a control group and observing the changes in the thermal threshold among the TLS group helped to interpret the thermal threshold findings in the study. Several factors such as sleep quality and patterns, psychosocial factors, pain medications, underlying medical history, caffeine intake, gender, age and body composition may influence sensory perception and outcomes of sensory testing. While few factors such as BMI, pain medication, underlying medical conditions were monitored and controlled in the trial, other factors were not controlled which could be potential confounders. As the NLSD + HP with BO was considered as an experimental intervention and the study was designed to test the design features and its effects, the study findings had limited external validity. However, the design and development of the novel lumbar support device had undergone strict scientific process of reliability and validity examination to ensure that it is functioning well before the current trial was conducted. In addition, the current trial encompassed a wide range of outcome measures which were relevant to current clinical practice in low back pain management and hence, the findings could be relevant to practice. Therefore, future studies evaluating the effects of the novel lumbar support might need to include these additional variables as important outcomes among CLBP patients. Another limitation of the study was that the participants recruited in this study showed only a minimal pain related disability with the Oswestry score showing $<20 \%$ in three of the four groups. Therefore, it was important to acknowledge that the effectiveness of the new device should be further investigated not only to assess its long-term clinical effects, but also its usefulness in low back patients presenting moderate and severe disability. Currently, a clinical trial is ongoing to determine long-term effects of the NLSD + HP with BO among CNLBP population and therefore any wider implications and effects of the novel lumbar support need among CNLBP patient needs to be revisited again.

\section{CONCLUSIONS}

The immediate effects of the novel lumbar support device showed potential beneficial effects on pain modulation and core muscle function among patients with CNLBP in comparison to the traditional lumbar support. Further clinical trial evaluating the long-term clinical effects of the novel lumbar support device is needed to understand the overall benefits of the device and its usefulness for people with CNLBP. 


\section{CONFLICT OF INTERESTS}

The authors declare that they have no conflict of interests.

\section{REFERENCES}

1. Dimer da Luz R, da Silva Santos M, Steffen Evaldt A, da Silva Matos L, Boff Daitx R, Döhnert MB. Neuromuscular electrical stimulation associated with core stability exercises in nonspecific postural low back pain: a randomized clinical trial. Muscles Ligaments Tendons J 2019;9(3);446-56.

2. De Giorgio A, Padulo J, Kuvačić G. Effectiveness of yoga combined with back school program on anxiety, kinesiophobia and pain in people with non-specific chronic low back pain: a prospective randomized trial. Muscles Ligaments Tendons J 2018;8(1);104-12.

3. Steffens D, Maher C, Pereira L, Stevens M, Oliveira V, Chapple M. Prevention of low back pain: a systematic review and meta-analysis. JAMA Intern Med 2016;176:199-208.

4. Pengel HM, Maher CG, Refshauge KM. Systematic review of conservative interventions for subacute low back pain. Clin Rehabil 2002;16(8):811-20.

5. Calmels P, Queneau P, Hamonet C, et al. Effectiveness of a lumbar belt in subacute low back pain: an open, multicentric, and randomized clinical study. Spine 2009;34(3):215-20.

6. van Duijvenbode I, Jellema P, van Poppel M, van Tulder M. Lumbar supports for prevention and treatment of low back pain. Cochrane Database Syst Rev 2008(2):CD001823.

7. van Poppel MN, de Looze MP, Koes BW, Smid T, Bouter LM. Mechanisms of action of lumbar supports: a systematic review. Spine 2000;25(16):2103-13.

8. McNair PJ, Heine PJ. Trunk proprioception: enhancement through lumbar bracing. Arch Phys Med Rehabil 1999;80(1):96-9.

9. Cholewicki J, McGill KC, Shah KR, Lee AS. The effects of a three-week use of lumbosacral orthoses on trunk muscle activity and on the muscular response to trunk perturbations. BMC Musculoskelet Disord 2010;11(1):1-10.

10. Morrisette DC, Cholewicki J, Patenge WF, Logan S, Seif G, McGowan S. A randomized clinical trial comparing extensible and inextensible lumbosacral orthoses and standard care alone in the management of lower back pain. Spine 2014;39(21):1733.

11. Fayolle-Minon I, Calmels P. Effect of wearing a lumbar orthosis on trunk muscles: study of the muscle strength after 21 days of use on healthy subjects. Joint Bone Spine 2008;75(1):58-63.

12. Dissanguan D, Sitilertpisan P, Joseph L, Paungmali A. Effectiveness of Lumbar Support in Management of Low Back Pain: A Systematic Review. Online J Health Allied Scs 2018;17(4):3.

13. Padulo J, Oliva F, Frizziero A, Maffulli N. Muscles, Ligaments and Tendons Journal - Basic principles and recommendations

in clinical and field Science Research: 2016 Update. Muscles Ligaments Tendons J 2016;6(1):1-5.

14. Dissanguan D, Sitilertpisan P, Kiatwattanacharoen S, Joseph LH, Puangmali P, Paungmali A. Reliability and Validity of the Feedback Sensor for Activating the Transversus Abdominis Muscle. Open Biomed Eng 2019;13(1):67-73.

15. Paungmali A, Joseph LH, Sitilertpisan P, Pirunsan U, Uthaikhup S. Lumbopelvic core stabilization exercise and pain modulation among individuals with chronic nonspecific low back pain. Pain Pract 2017;17(8):1008-14.

16. Joseph LH, Hancharoenkul B, Sitilertpisan P, Pirunsan U, Paungmali A. Effects of massage as a combination therapy with Lumbopelvic stability exercises as compared to standard massage therapy in low Back pain: a randomized Cross-over study. Int J Ther Massage Bodywork 2018;11(4):16.

17. Litcher-Kelly L, Martino SA, Broderick JE, Stone AA. A systematic review of measures used to assess chronic musculoskeletal pain in clinical and randomized controlled clinical trials. J Pain 2007;8(12):906-13.

18. Malmström E-M, Stjerna J, Högestätt ED, Westergren H. Quantitative sensory testing of temperature thresholds: Possible biomarkers for persistent pain? J Rehabil Med 2016;48(1):43-7.

19. Leonard J, Paungmali A, Sitilertpisan P, Pirunsan U, Uthaikhup S. Changes in Transversus Abdominis Muscle Thickness after Lumbo-Pelvic Core Stabilization Training among Chronic Low Back Pain Individuals. Clin Ter 2015;166(5):e312-6.

20. Cohen J. Statistical power analysis for the behavioural sciences: London: Academic Press, 1977.

21. Lehmann JF, Warren CG, Scham SM. Therapeutic heat and cold. Clin Orthop Relat Res 1974;99:207-45.

22. Walk D, Sehgal N, Moeller-Bertram T, et al. Quantitative sensory testing and mapping: a review of nonautomated quantitative methods for examination of the patient with neuropathic pain. Clin J Pain 2009;25(7):632-40.

23. Neziri AY, Curatolo M, Limacher A, et al. Ranking of parameters of pain hypersensitivity according to their discriminative ability in chronic low back pain. Pain 2012;153(10):2083-91.

24. Nadler SF, Steiner DJ, Petty SR, Erasala GN, Hengehold DA, Weingand KW. Overnight use of continuous low-level heatwrap therapy for relief of low back pain. Arch Phys Med Rehabil 2003;84(3):335-42.

25. Rackwitz B, de Bie R, Limm H, von Garnier K, Ewert T, Stucki G. Segmental stabilizing exercises and low back pain. What is the evidence? A systematic review of randomized controlled trials. Clin Rehabil 2006;20(7):553-67. 